\title{
Measurement does not always aid state discrimination
}

\author{
Kieran Hunter \\ Department of Physics, University of Strathclyde, Glasgow G4 ONG, Scotland
}

\begin{abstract}
We have investigated the problem of discriminating between nonorthogonal quantum states with least probability of error. We have determined that the best strategy for some sets of states is to make no measurement at all, and simply to always assign the most commonly occurring state. Conditions which describe such sets of states have been derived.
\end{abstract}

PACS numbers: 03.67.-a

Finding the best measurement strategy to perform on some quantum signal is an important problem, with particular relevance to quantum communication and computation. The optimal measurement strategy will depend on the purpose of the measurement. To optimise our strategy we must define a figure of merit function which provides a measure of the appropriateness of a strategy. Then we must find the measurement which maximises (or minimises) the chosen figure of merit.

A commonly considered example of this is the probability of incorrectly identifying the state $P_{e}$. A set of necessary and sufficient conditions for a measurement to minimise $P_{e}$ (or more generally the mean Bayes cost) are known [1, 2, 3], but a solution to these conditions has only been found in a small number of cases. These are: when there are only two possible states of the signal [1], when the states are linearly independent [4], when the states are equiprobable and sum to the identity [3], the cases of equiprobable symmetric [5] (or geometrically uniform [6]) and multiply symmetric [7] states, and the case of three mirror symmetric qubit states [8].

In this paper we present an additional solution to these minimum error conditions. We describe sets of states where no measurement discriminates between the states better than assigning the a priori most likely state to the signal.

To find an optimal strategy we describe the measurement by its Probability Operator Measure (POM) elements $\hat{\Pi}_{k}$. These POM elements are operators which represent the probability of occurrence of each possible outcome of a measurement. The probability $P(k \mid j)$ of the outcome $k$ occurring given that the system was in its $j^{\text {th }}$ state $\hat{\rho}_{j}$ is

$$
P(k \mid j)=\operatorname{Tr}\left(\hat{\Pi}_{k} \hat{\rho}_{j}\right)
$$

For the POM elements $\hat{\Pi}_{k}$ to represent probabilities, they must be subject to the following constraints:

1. All the $\hat{\Pi}_{k}$ 's are Hermitian,

2. Their eigenvalues non-negative,

3. The total probability of all outcomes for any input sums to 1:

$$
\sum_{k} \hat{\Pi}_{k}=\hat{1}
$$

The conditions for a measurement strategy $\left\{\hat{\Pi}_{k}\right\}$ to minimise the probability of erroneously identifying the state of the signal can be easily derived from those for minimising the mean Bayes cost [1] by an choosing the cost of being wrong to be a constant. The minimum error conditions can then be stated as

$$
\sum_{j} \hat{\Pi}_{j} p_{j} \hat{\rho}_{j}-p_{k} \hat{\rho}_{k} \geq 0 \forall k,
$$

which means that the operator on the left is both Hermitian and positive semidefinite. From this can be derived the necessary condition [1]

$$
\left(\sum_{j} \hat{\Pi}_{j} p_{j} \hat{\rho}_{j}-p_{k} \hat{\rho}_{k}\right) \hat{\Pi}_{k}=0 \forall k,
$$

which implies the Hermiticity of (3).

The derived condition (4) has an interesting property. It is always satisfied by the POM

$$
\hat{\Pi}_{j}=\hat{1}, \hat{\Pi}_{k \neq j}=\hat{0} .
$$

This POM corresponds to not making any measurement on the signal at all, and simply always assigning the state $\hat{\rho}_{j}$ to the signal. In effect this POM is a consistent guessing strategy. This POM automatically makes the left side of (3) Hermitian.

When such a POM is used, the condition (3) becomes

$$
p_{j} \hat{\rho}_{j}-p_{k} \hat{\rho}_{k} \geq 0 \forall k,
$$

for some value of $j$. When this condition is satisfied by the set of possible states of the signal $\left\{\hat{\rho}_{k}\right\}$ with prior probabilities $p_{k}$, there exists no measurement which distinguishes the states better than guessing. We must now determine the physical meaning of (6).

It is obvious that the state $\hat{\rho}_{j}$ must be the most likely state. This can be verified be taking the trace of (6), which gives $p_{j} \geq p_{k} \forall k$. It is also clear that $\hat{\rho}_{j}$ cannot be a pure state (except in the trivial case where all of the states are identical) since then the operator on the left of (6) would have a negative expectation value for some states. Indeed this tells us that it is not sufficient for the state $\hat{\rho}_{j}$ to be mixed: this state must span the entire space of the states $\hat{\rho}_{k}$. These are necessary, but not sufficient for (6) to be satisfied. 
Denoting the most likely state as $\hat{\rho}_{0}$, we can restate the condition (6) as the vector inequality "In every direction, the component of the operator $p_{0} \hat{\rho}_{0}$ must be greater than or equal to the components of each of the operators $p_{k} \hat{\rho}_{k}$ in that direction.":

$$
\left\langle\phi\left|\left(p_{0} \hat{\rho}_{0}-p_{k} \hat{\rho}_{k}\right)\right| \phi\right\rangle \geq 0 \forall k,|\phi\rangle .
$$

If all of the states other than the most likely state $\hat{\rho}_{0}$ are pure states, $\hat{\rho}_{k}=\left|\psi_{k}\right\rangle\left\langle\psi_{k}\right|$, we can simplify this condition by noting that the only significant vector $|\phi\rangle$ in this case is $|\phi\rangle=\left|\psi_{k}\right\rangle$. The condition then reduces to

$$
\left\langle\psi_{k}\left|p_{0} \hat{\rho}_{0}\right| \psi_{k}\right\rangle \geq p_{k} \forall k .
$$

If instead the $\hat{\rho}_{k}$ are mixed we shall obtain one such relation for each pure state that $\hat{\rho}_{k}$ can be decomposed into, with $p_{k}$ being multiplied by the weight of that pure state in $\hat{\rho}_{k}$.

This simplifies even further if the most likely state $\hat{\rho}_{0}$ is a no-information (maximally mixed) state, $\hat{\rho}_{0}=\frac{1}{D} \hat{1}$ where $D$ is the dimension of the state space:

$$
\frac{p_{0}}{D} \geq p_{k} \forall k .
$$

The best way to illustrate the significance of these states is by an example.

Let us consider a communication channel in which the signal can be any one of $N$ pure states $\left|\psi_{k}\right\rangle$. The preparation of each of the $N$ states are equally likely, but there is also a chance that the preparation will fail and a completely random state will be sent. Here we are viewing the preparation to be both the transmitter and the channel itself, as it does not matter where these failures occur. We wish to identify with least probability of error what was sent: either a specific signal state or a failed transmission. Is there any point in measuring the received signal?

In this example the only state which could satisfy the requirement to span the space is the 'failed transmission'. Since the signal is completely random in this case the only state which can be assigned to the signal is $\hat{\rho}_{0}=\frac{1}{D} \hat{1}$, where $D$ is the dimension of the space.

Since the $N$ possible signals are equally likely, we can set their probabilities $p_{k}=p=\frac{1-p_{0}}{N}$. The simplified condition for the case of discriminating unlikely pure states from a single maximally mixed state (9) then gives

$$
\frac{p_{0}}{D} \geq \frac{1-p_{0}}{N}
$$

which implies

$$
p_{0} \geq \frac{D}{D+N}
$$

If this inequality holds then there is no measurement which will distinguish the signal states from a failed transmission.

For the case of three qubit signal states this would give $p_{0} \geq \frac{2}{5}$. Even at a failure rate of only two fifths for these signal states, it is still impossible to find any measurement which discriminates the signal states and failure with less probability of error than always assuming that the preparation has failed.

At this point it is worth discussing what this result means, and its limitations. The conditions on the set of states such that the no measurement POM is optimal have a clear interpretation in terms of the likelihood of the states. To understand this we must look at how the measurement affects the assignment of the signal state.

Before we measure the state of the signal the only information we have about that state are the prior probabilities of each possible state. Thus we assign these prior probabilities as the likelihood of detecting each state, with the highest probability belonging to the most likely state. Once we have measured the state we also know the measurement outcome. We can use Bayes rule with (11) and $p_{j}$ to calculate the probability $P(j \mid k)$ that the signal state was $\hat{\rho}_{j}$ given that the measurement outcome was k. These $P(j \mid k)$ are the probabilities we assign to each state on the basis of our updated information which now includes the result of our measurement.

If the a priori most likely state will remain the most likely state whatever the result of any measurement made, then no measurement discriminates between the states better than guessing. This would not be a surprising result if it were not for the fact that it is quite easy to obtain such a set of states. It can also hold for some classical systems, if there is a severe restriction on the form of the measurements which can be made. Only using quantum systems and measurements can we say that it can be satisfied for all physically possible measurements.

Just because we have established that we cannot identify the most likely state by a measurement does not mean that there is no point in performing a measurement. One could, for example, try to identify the next most likely state. In the communication example given earlier, we would forget about trying to determine if the signal was real or a failed transmission and instead ask "If we assume that this signal is not a failed transmission, what is the most likely state of the signal?" This will determine which of the $N$ signal states is the most likely to have been transmitted, but it would still be more likely that transmission failed.

We can also give up on any positive identification of the state and instead try to obtain as much information as possible about the signal. The appropriate figure of merit for this would be the mutual information gain $I$ [9, 10, 11] which is always positive for any actual measurement and zero for our no measurement POM. That this gives a different result should not be surprising as the maximum information strategy is different from the minimum error strategy even for very simple examples [12].

In conclusion, we have found an interesting solution to the problem of discriminating between the possible states of a quantum signal or system with least probability of error. For some sets of states it is possible to satisfy the necessary and sufficient conditions for minimum er- 
ror by not making any measurement at all, and simply assigning the most common state to the system. We have explored the restrictions on such sets of states, and developed simplifications for these when the most likely state is maximally mixed and also when the other states are pure states.

These results were illustrated by a quantum communi- cations example, and can be easily interpreted in terms of Bayes rule.

This work was supported by the UK Engineering and Physical Sciences Research Council. The author would like to thank Professor Stephen M. Barnett for his helpful advice on the production of this paper.
[1] C.W. Helstrom, Quantum Detection and Estimation theory (New York: Academic 1976).

[2] A.S. Holevo, Probabilistic and Statistical Aspects of Quantum Theory (Amsterdam: North-Holland 1982).

[3] H.P. Yuen, R.S. Kennedy, M. Lax, IEEE Trans. Inf. Theory IT-21 125 (1975).

[4] R.S. Kennedy, M.I.T. Res. Lab. Electron. Quart. Progr. Rep. 110142 (1973).

[5] M. Ban, K. Kurokawa, R. Momose, O. Hirota, Int. J. Theor. Phys. 361269 (1997).

[6] Y.C. Eldar, G.D. Forney Jr, IEEE Trans. Inf. Theory 47 858 (2001).
[7] S.M. Barnett, Phys. Rev. A 64 030303(R) (2001).

[8] E. Andersson, S.M. Barnett, C.R. Gilson, K. Hunter, Phys. Rev. A. 65, 052308 (2002).

[9] E.B. Davies, IEEE Trans. Inf. Theory IT-24 596 (1978).

[10] M. Sasaki, S.M. Barnett, R. Jozsa, M. Osaki and O. Hirota, Phys. Rev. A. 593325 (1999).

[11] J. Mizuno, M. Fujiwara, M. Akiba, T. Kawanishi, S.M. Barnett, M. Sasaki, Phys. Rev. A. 65, 012315 (2002).

[12] R.B.M. Clarke, V.M. Kendon, A. Chefles, S.M. Barnett, E. Riis, M. Sasaki, Phys. Rev. A. 64012303 (2001). 